\title{
Pharmakologische Stoffwechselbeeinflussung zur Verlängerung der Wiederbelebungszeit bei Sauerstoffmangel in Normothermie
}

\author{
Rolf Voss und Hans R. Schoen \\ Chirurgische Klinik der Justus Liebig-Universität, Gießen
}

\begin{abstract}
Pharmacological effects on metabolism aiming at a prolongation of revival time under oxygen lack in normothermy. The basal metabolic rate depends on the level of circulating thyroid hormones. A close correlation exists between the duration of the revival time (beginning of ischemia until appearance of irreversible damages) of an organ or the organism and the intensity of metabolism. For this reason revival time depends also upon the level of thyroid hormones. By restraining the thyroid activity through premedication in the form of inorganic iodine (Endojodin $\left({ }^{B}\right)$, we obtained a prolongation of the duration of the revival time in normothermic rabbits and dogs under the conditions of asphyxia and hypoxemia. This effect was increased when a pyrimido-pyrimidine derivative (Dipyridamol $=$ Persantin (8) was added, which potentiates the vasodilatator effect of adenosine. By means of this combined premedication, we found that the breakdown of the high-energy phosphates in the brain and myocardium occurred more slowly and that - after a 10-minute-long inflow occlusion - the effect of hypoxemia in dog hearts proved to cause a less rapid increase of the concentration of lactate, pyruvate and inorganic phosphate. Electron-microscopic investigations performed in the papillary muscle of the heart of asphyctic rabbits showed that certain asphyxial damages could be successfully delayed or at least reduced with our combined premedication, which is probably due to an improvement of energy utilization during anaerobiosis. The duration of the circulatory standstill, caused by inflow occlusion, could be extended from 8 up to 15 minutes in normothermic dogs.
\end{abstract}

\section{EINLEITUNG}

Die Höhe des Schilddrüsenhormonspiegels ist unter Berücksichtigung des Faktors Zeit (t) direkt proportional der Höhe des Basalstoffwechsels (Scharf 1963). Diese Proportionalität ist nur bei einem Adrenalindefizit gestört.

Hyperthermie und Hyperthyreose einerseits und Hypothermie und Hypothyreose andrerseits gleichen sich bezüglich ihres Energieverbrauches. BRETSCHNEIDER (1961) hat das Thyroxin als Hypothermie-Antagonisten bezeichnet. Wir sprechen hier vom Thyroxin pars pro toto für das ganze Spektrum der Schilddrüsenhormone. Nach pharmakologischer Drosselung der Thyroxinproduktion ist mit einem geringeren Energieumsatz und entsprechend kleinerem $\mathrm{O}_{2}$-Verbrauch zu rechnen. Daher muß sich unter dieser Voraussetzung ein $\mathrm{O}_{2}$-Mangel weniger deletär auswirken. 
Bei plötzlicher Unterbrechung der Blutzufuhr (Ischaemie) treten die Funktionsstörungen an einem Organ in typischer Reihenfolge auf (SCHNEIDER 1958): 1. P hase: Latenzzeit oder Zeit der noch ungestörten Funktion. 2. Ph as e : Zeit vom Ischaemicbeginn bis zum Ausfall der geprüften Funktion. Sie wird Überlebenszeit (ÜBZ; survival time) oder maximale Funktionszeit genannt und beträgt für Gehirn oder Niere nur 8 bis 10 Sekunden. 3. Phase: Zeitspanne vom Beginn der Ischaemie bis zum Eintritt irreversibler Schädigungen. Sie wird als Wiederbelebungszeit bezeichnet (WBZ; revival time) und ist nur retrospektiv (von der Funktion her) zu bestimmen. Die WBZ des Gesamtorganismus liegt in Normothermie - begrenzt durch die WBZ des Herzens - bei 4 bis 5 Minuten, für das Gehirn bei 8 bis 10 Minuten und die Niere bei 2 bis 3 Stunden.

Obwohl diese Definitionen strenggenommen nur für die Ischaemie gelten, haben wir sie auch bei der Besprechung und Deutung unserer Asphyxie- und Hypoxieversuche benutzt.

Verwendete Abkürzungen: UBZ: Überlebenszeit, WBZ: Wiederbelebungszeit, HVL: Hypophysenvorderlappen, SD: Schilddrüse, PKr: Phosphokreatin, ATP, ADP, AMP: Adenosin-5'-tri-(di-, mono-)phosphat, $\mathrm{P}_{\mathrm{a}}$ : anorganisches Phosphat, BTS: Brenztraubensäure (Pyruvat), MS: Milchsäure (Lactat).

\section{ERGEBNISSE}

Unsere Tierversuche wurden mit dem Ziel angestellt, die UBZ und WBZ des Herzens und Gesamtorganismus in Normothermie zu verlängern. Die Verlängerung von UBZ und WBZ hat heute für die Klinik und speziell für die Herz- und Gehirnchirurgie und die Transplantation ganzer lebenswichtiger Organe große Bedeutung.

Durch chemische Analysen der für den Energiestoffwechsel wichtigen Substrate PKr, ATP, ADP, AMP, $P_{a}$, BTS und MS versuchten wir retrospektiv Aufschluß über die Dauer von $\mathrm{OBZ}$ und WBZ zu erhalten, wenn die Tiere infolge der Versuchsanordnung das Experiment nicht überleben konnten. Bei Versuchen, die auf ein Überleben der Kreislaufunterbrechung angelegt waren, wurde die Dauer von ÜBZ und WBZ direkt bestimmt.

Auf Grund der Untersuchungen von Kuschinsky (1933) und Loeser (1934) wissen wir, daß nach Gabe von 0,5 bis $10,0 \mathrm{mg} / 100 \mathrm{~g}$ anorganischem Jod der Grundumsatz um etwa $20 \%$ gesenkt wird. Durch Zufuhr von anorganischem Jod wird hier die physiologische Höhe des Plasmajodspiegels, der unterhalb von 20 bis $30 \% \%$ die Funktion der Schilddrüse gewährleistet, überschritten und der Hypophyse eine erhöhte Thyroxinkonzentration vorgetäuscht, die den Hypophysenvorderlappen veranlaßt, die Thyreotropinausschüttung einzuschränken (Täuschungseffekt). Der HVL ist nicht in der Lage, das Thyroxinmolekül in seiner Gesamtheit zu registrieren. Aus Untersuchungen von Kuschinsky (1933), Loeser. (1964), Scharf, Ehrengrand \& Förster (1954) ergab sich, daß die Adenohypophyse einen spezifischen Rezeptor für den Plasmajodspiegel besitzt. VON EULeR \& HoLmgren (1956) konnten mit Hilfe gezielter lokaler Mikroinjektionen von Thyroxin in die Hypophyse des Kaninchens nachweisen, daß allein dem HVL diese Jodsensibilität zukommt. 


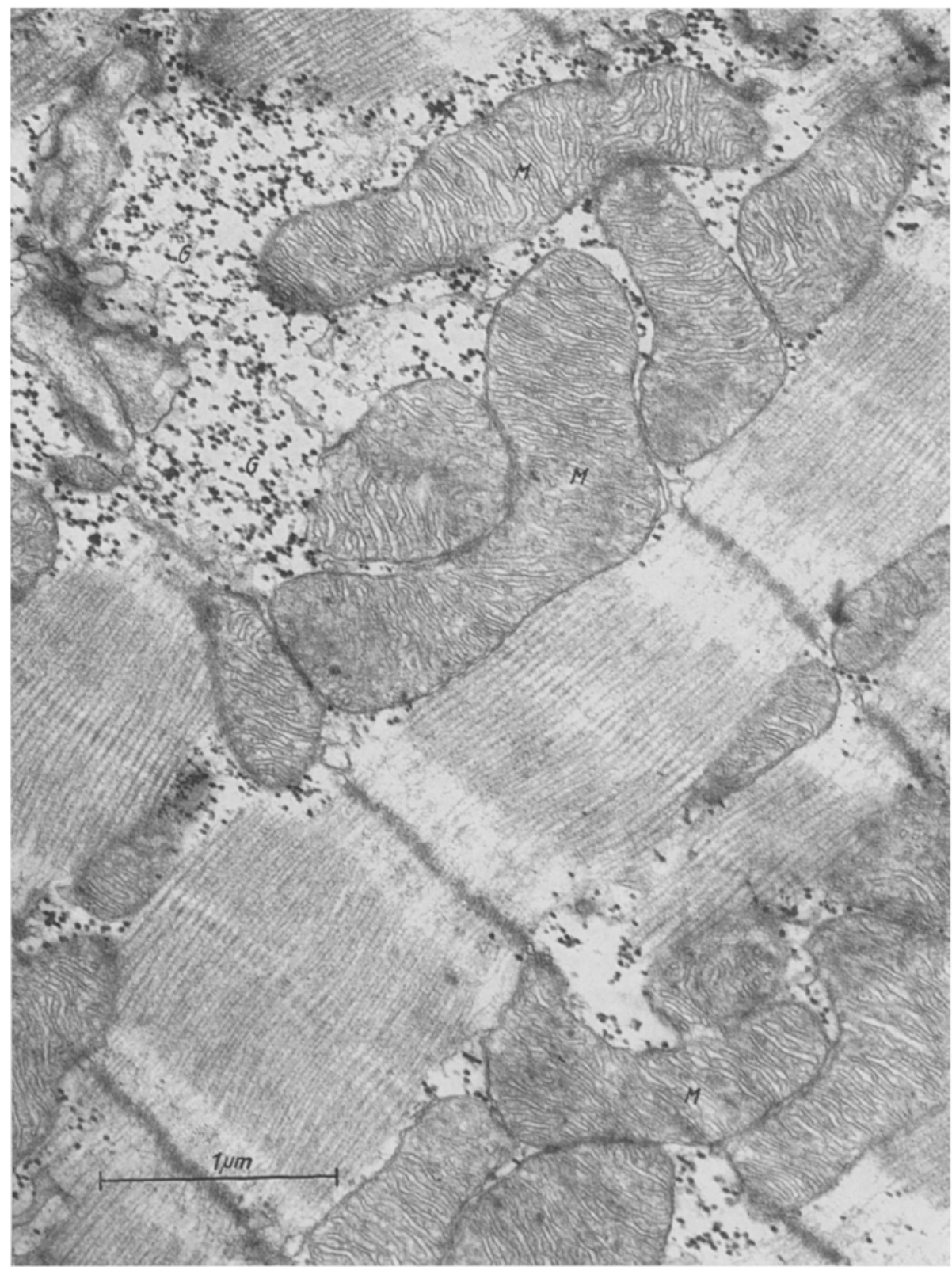

Abb. 1: Papillarmuskel eines Kaninchens (Normaltier). Zwischen den Myofibrillen didht liegende große Mitochondrien (M). Im Inneren derselben zahlreiche Cristae mitochondrales. Unmittelbar neben den Mitochondrien kontrastreiche Glykogengranula (G) einheitlicher GröBe und Gestalt. $30000: 1$. (Nach Vogell et al. 1964) 


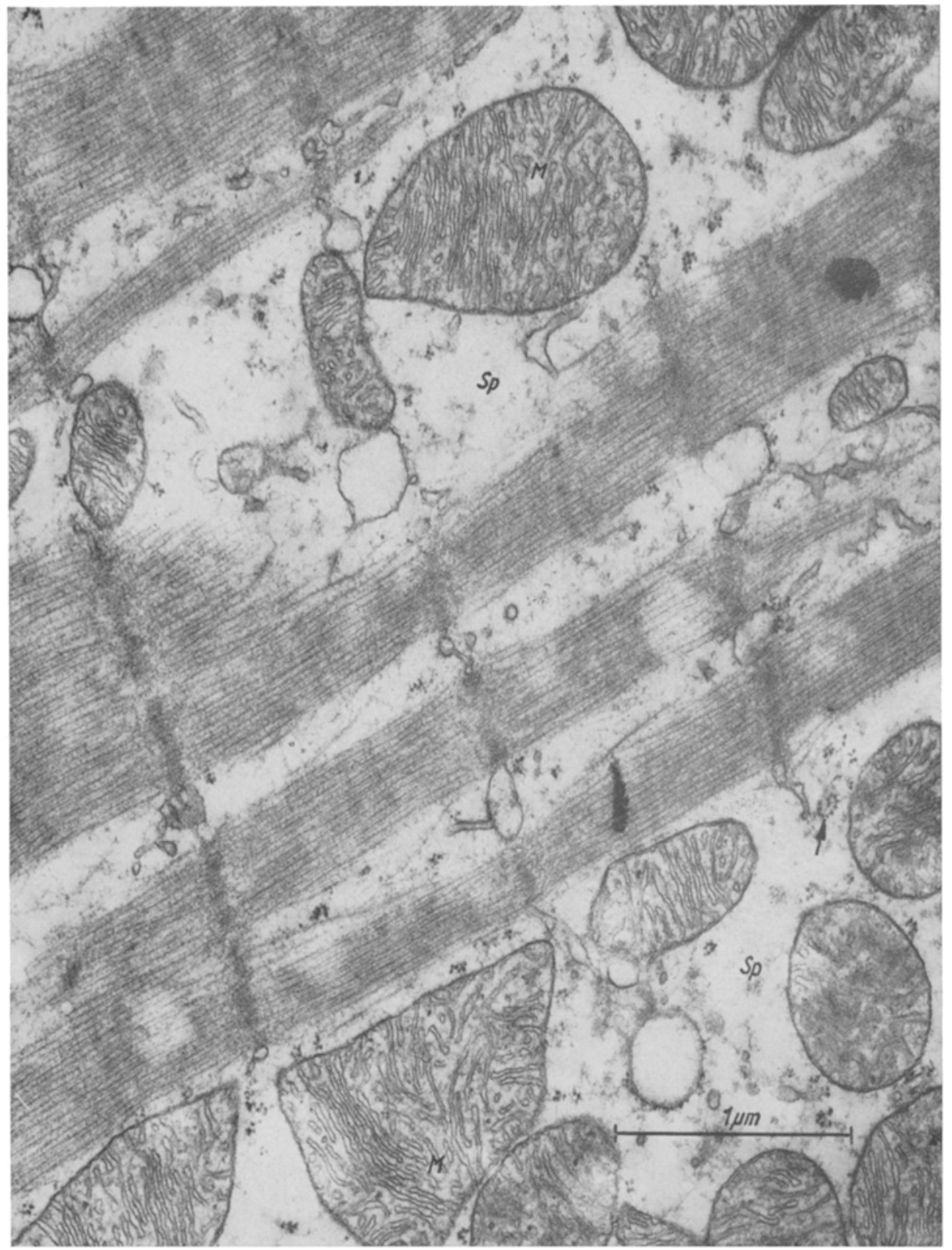

Abb. 2: Papillarmuskel nach 3 Minuten Asphyxie. Die Glykogengranula sind praktisch verschwunden. Die Mitochondrien sind gequollen. Die Struktur der Membran ist unverändert. Die Erweiterung der interfibrillären Spalten ist wahrscheinlich auf ein beginnendes hypoxämisches Ódem zurückzuführen. $30000: 1$. (Nach VagelL et al. 1964) 


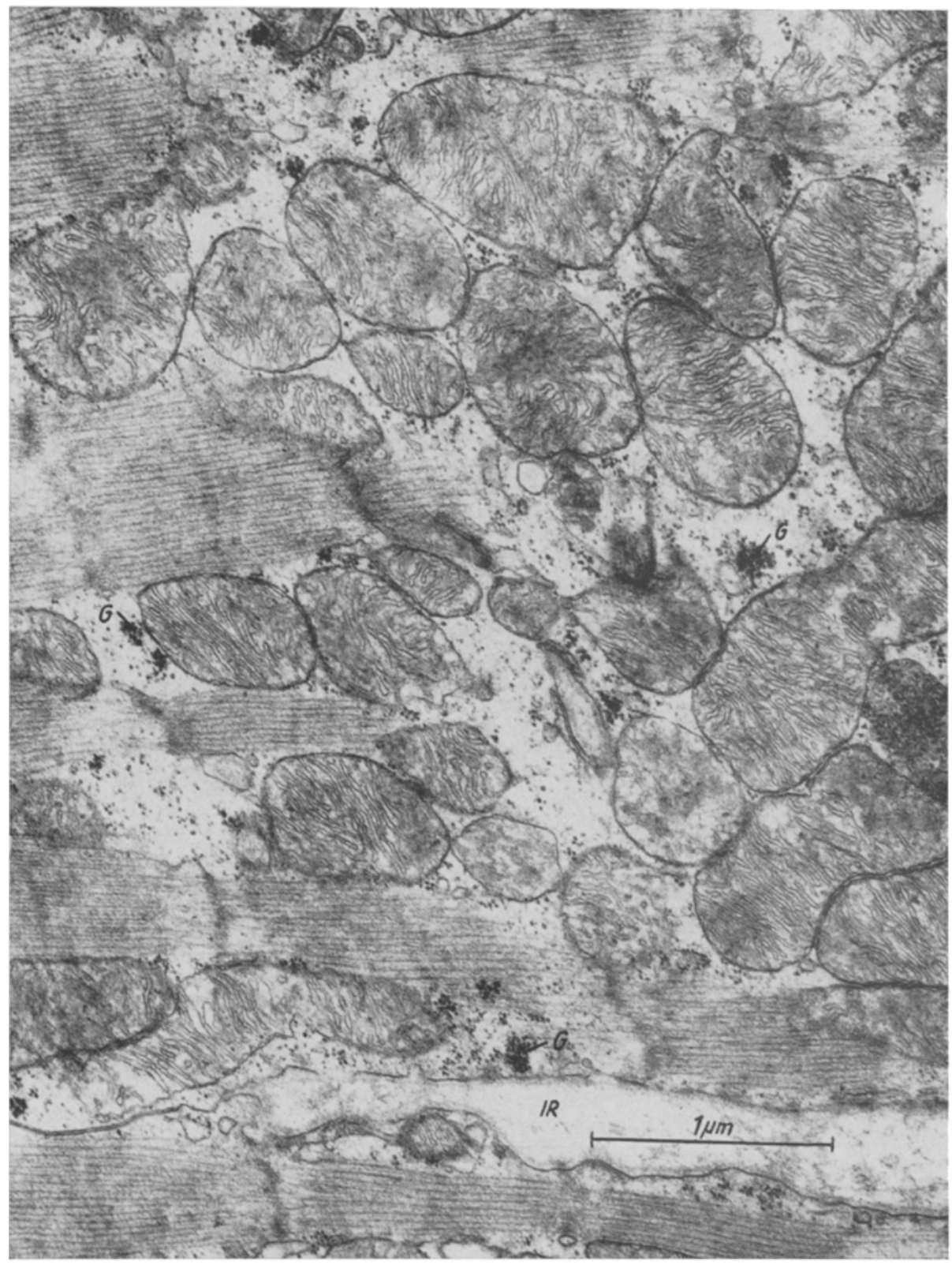

Abb. 3: Papillarmuskel nach 3 Minuten Asphyxie mit Prädemikation von $80 \mathrm{mg}$ Jod pro kg Körpergewicht 12 Stunden vor Versuchsbeginn. Im Gegensatz zu Abbildung 2 sind Komplexe glykogenhaltiger Granula nachweisbar $(G)$. Ein interfibrilläres Odem scheint nicht vorzuliegen. Die Mitochondrien zeigen keine merklichen Veränderungen. $30000: 1$.

(Nach Voget. et al. 1964) 


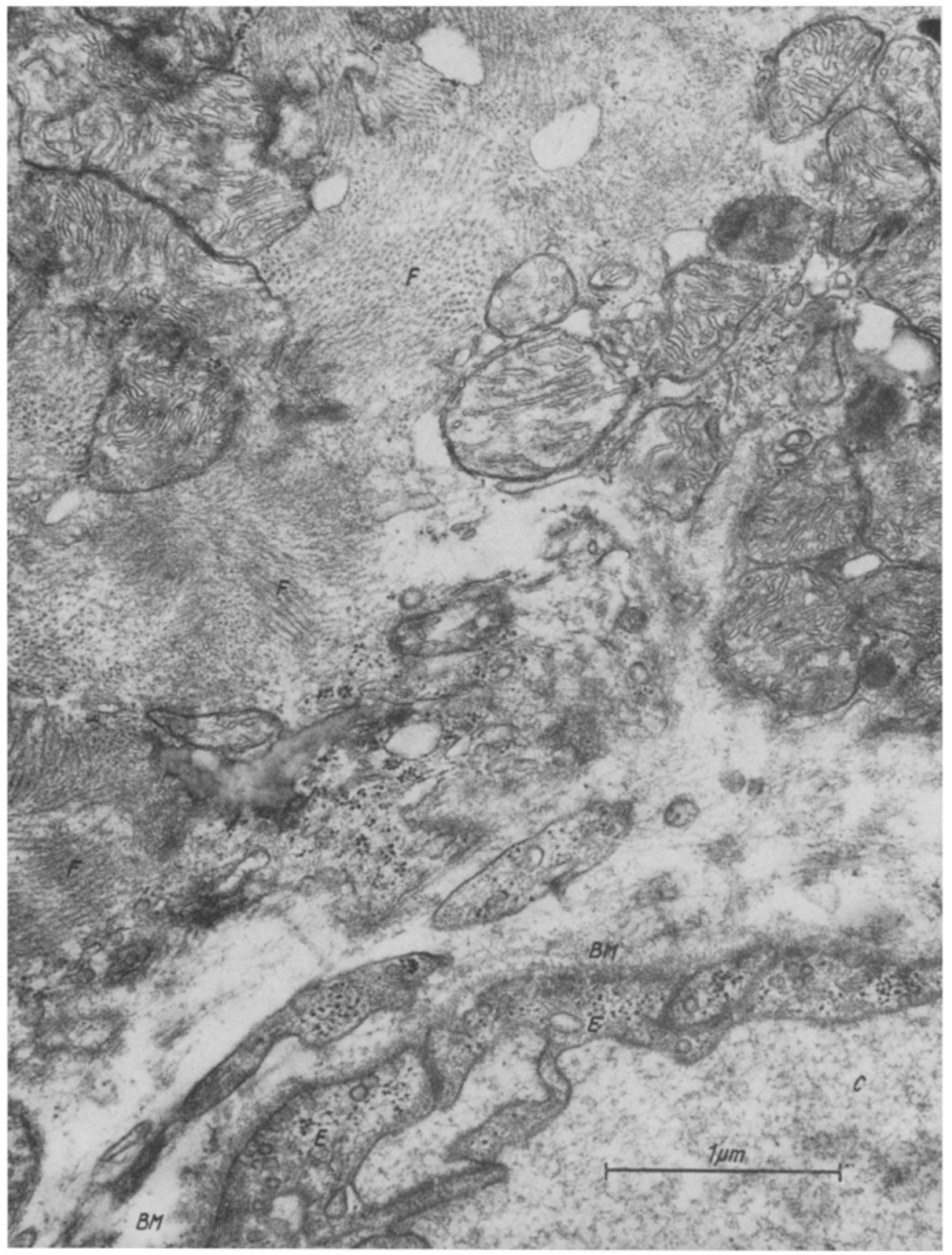

Abb. 4: Papillarmuskel nach 15 Minuten Asphyxie ohne Prämedikation. Im unteren Bildrand rechts Anschnitt einer Kapillare (C), in deren Umgebung die Anzeichen des Odems zu erkennen sind. Das die Muskelfaser gegen die Basalmembran (BM) abgrenzende Sarkolemm ist nicht nachweisbar. Glykogenhaltige Granula vereinzelt, vor allem im Kapillarendothel (E) nachweisbar, jedoch keine Zytopempsisbläschen, die Ausdruck eines Stoffaustausches zwischen Kapillare und Zellsystem sind (Moore \& Ruska 1957). Die mitochondrale Matrix ist gequollen, die Hüllmembran der Mitochondrien nicht immer geschlossen. Die Anordnung der Myofilamente (F) in den Fibrillen ist unregelmäßig und läßt keine einheitiche Verlaufsform erkennen. $30000: 1$. (Nach Vogeli et al. 1964) 


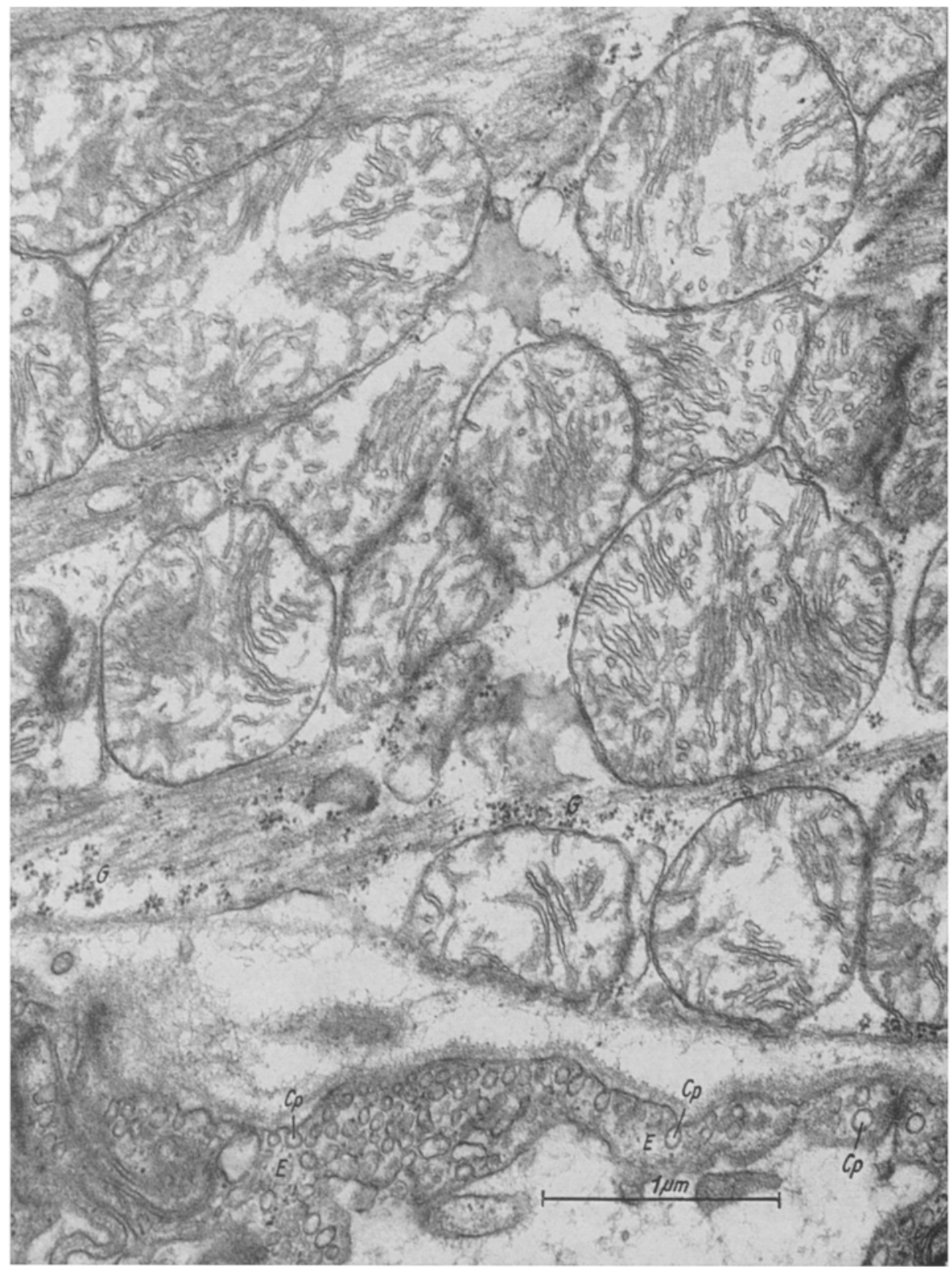

Abb. 5: Papillarmuskel nach 15 Minuten Asphyxie mit Prämedikation von $80 \mathrm{mg}$ Jod pro $\mathrm{kg}$ Gewicht 12 Stunden vor Versuchsbeginn. Das periphere Odem ist weniger ausgeprägt. Im Endothel (E) noch zahlreiche Zytopempsisbläschen (Cp). Glykogenhaltige Granula vor allem an der Faseroberfläche. Die mitochondrale Matrix ist deutlich gequollen. Die Hüllmembran der Mitochondrien ist hier jedoch in allen Fällen vorhanden. $30000: 1$. (Nach VoGkLL et al. 1964) 


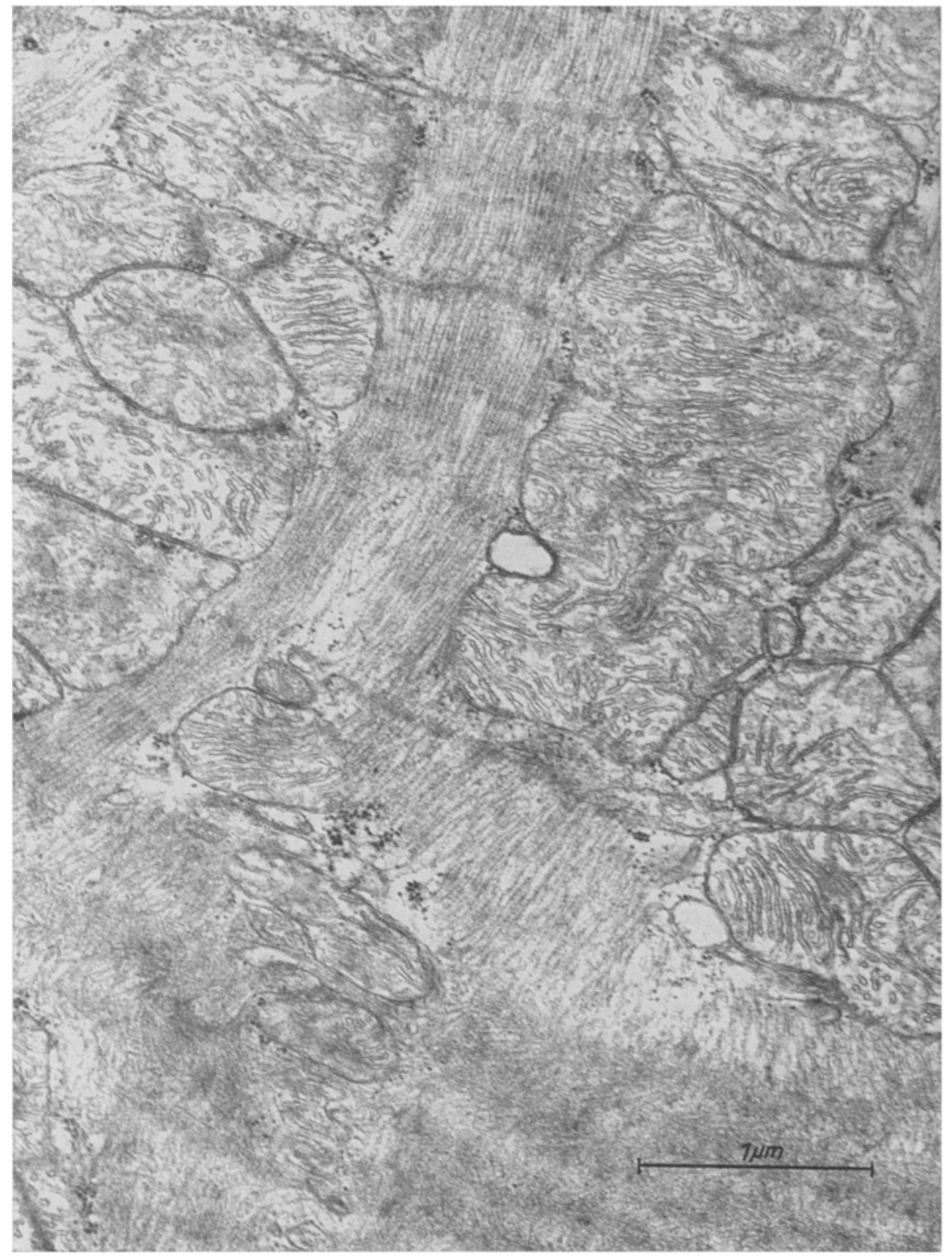

Abb. 6: Papillarmuskel des Kaninchens nach 6 Minuten Asphyxie ohne Prämedikation. Die glykogenhaltigen Granula sind deutlich vermindert. Die mitochondrale Matrix ist aufgehellt und gequollen. Infolge der dadurch bedingten Volumenvergrößerung der Mitochondrien ist das interfibrilläre Odem nur scheinbar weniger ausgeprägt. $30000: 1$ 


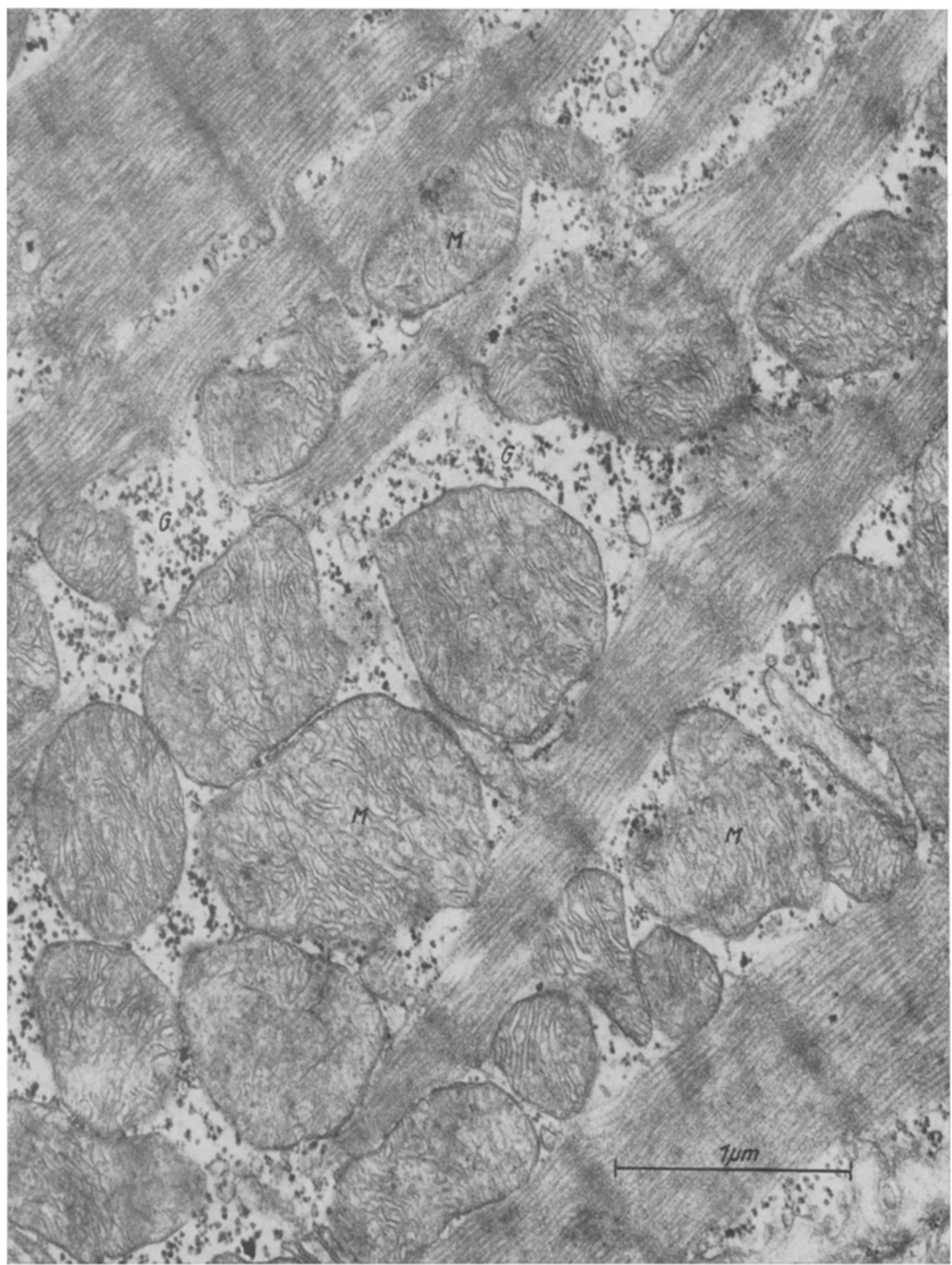

Abb. 7: Papillarmuskel nach 6 Minuten Asphyxie. Prämedikation $80 \mathrm{mg} / \mathrm{kg}$ Gewicht Jod 12 Stunden und $40 \mathrm{mg} / \mathrm{kg}$ Gewicht Persantin $1 / 2$ Stunde vor Versuchsbeginn. Im Vergleidh zum Normaltier sind nur unwesentliche Veränderungen feststellbar, die sich vor allem auf die Wiedergabe der hier vollständig kontrahierten Myofibrillen beziehen; Glykogengranula (G) und die Form der Mitochondrien (M) sind gegenüber dem Normalbild unverändert 
Das Hypophysen-Schilddrüsen-System stellt einen Reglerkreis dar; die Regelgröße ist der Basalstoffwechsel. Das anorganische Jod entfaltet seine Wirkung über zwei Stationen dieses Kreises: einmal über den Regler HVL, der die Produktion von Thyreotropin vermindert, zum andern über das Stellglied SD, in welchem eine Hemmung der Fermente Jodase und Jodinase eintritt. Die Stoffwechselimpulse aus der Körperperipherie, die über das Zwischenhirn zum Regler HVL gelangen, werden von

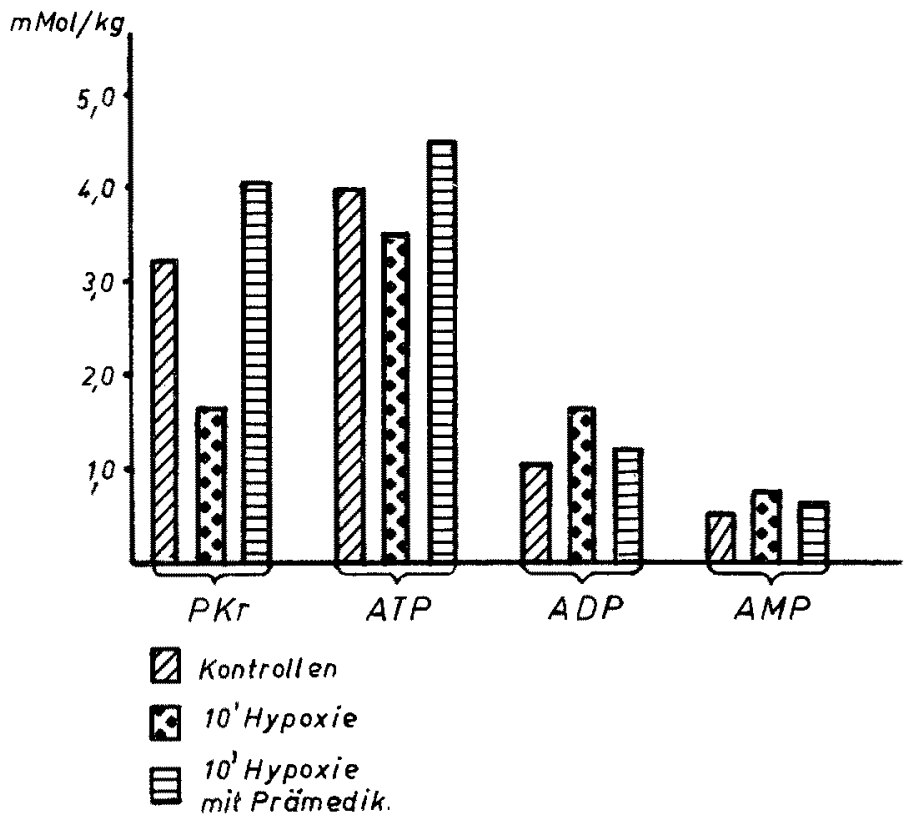

Abb. 8: Analyse der energiereichen Phosphate im linken Herzventrikel des Hundes. Veränderungen im Gehalt der energiereichen Phosphate unter verschiedenen Versuchsbedingungen. Das Phosphokreatin der prämedizierten Gruppe liegt noch über der Konzentration, die bei den Kontrolltieren, die keiner Hypoxie ausgesetzt wurden, gefunden wurde. Der Konzentrationsunterschied zur nicht prämedizierten Hypoxie-Gruppe ließ sich statistisch sichern

(WILCOXON-Test)

diesem infolge des Täuschungseffektes, den das anorganische Jod hervorruft, nicht mit einer Mehrproduktion von Thyretropin beantwortet. Da der Täuschungseffekt zeitlich begrenzt ist, kann anorganisches Jod nur wenige Tage stoffwechselsenkend wirken.

Unsere Stoffwechseluntersuchungen mit anorganischem Jod führten wir an $\mathrm{Ka}$ ninchenherzen durch. Die Tiere erhielten 14 Stunden vor Versuchsbeginn $75 \mathrm{mg} / \mathrm{kg}$ anorganisches Jod (Endojodin ${ }^{\circledR}$; Hersteller: Farbenfabriken BAYER, Leverkusen) intravenös appliziert. Tagesrhythmische Stoffwechselschwankungen wurden berücksichtigt.

Durch diese Jodprämedikation konnte der Glykogengehalt des Myocards um etwa $40 \%$ gesteigert werden. Die Tiere wurden danach durch Abklemmung der freipräparierten Trachea einer Asphyxie von 3 Minuten Dauer ausgesetzt. Beim Kontrollkollektiv sank der Glykogengehalt um $57 \%$ ab, im Gegensatz dazu betrug der Glykogenverlust beim prämedizierten Kollektiv nur $44 \%$. Das unterschiedliche Ver- 
halten des Glykogenverlustes nach 3minutiger Asphyxie war statistisch zu sichern. Entsprechend verhielt sich der Anstieg der Milchsäure. Die Jodprämedikation bewirkte offensichtlich eine Verlangsamung der anaeroben Glykolyse während des Sauerstoffentzugs.

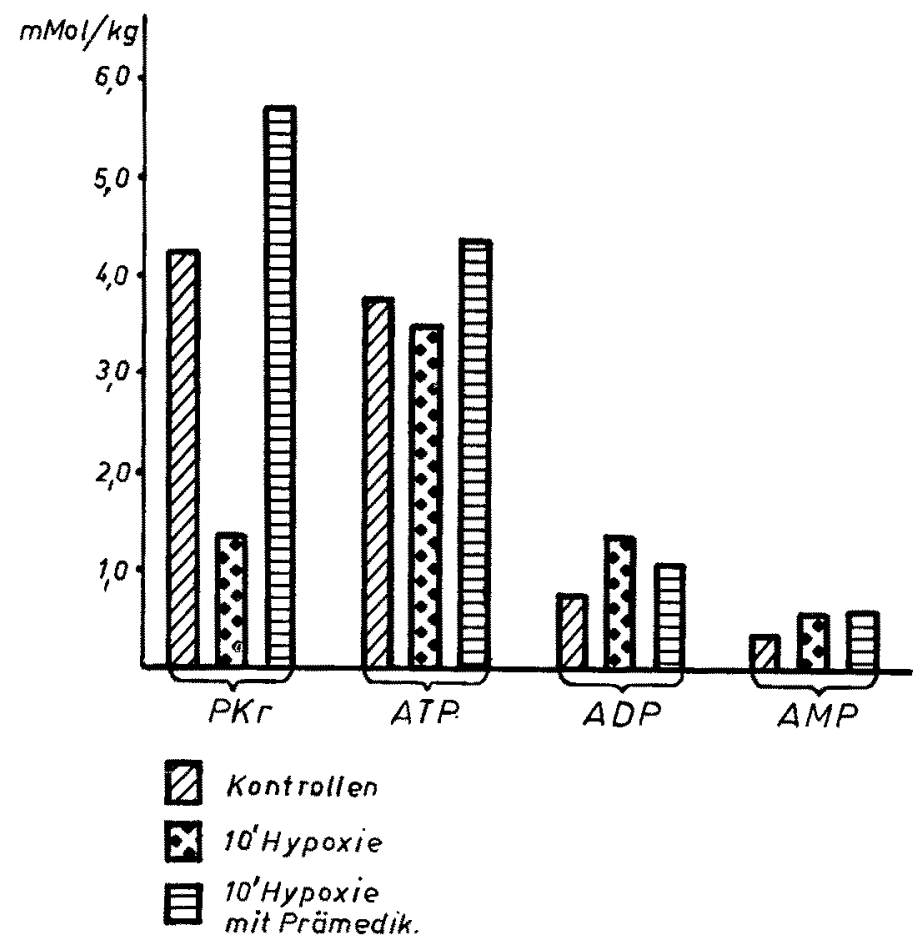

Abb. 9: Analyse der energiereichen Phosphate im rech te n Herzventrikel des Hundes. Veränderungen im Gehalt der energiereichen Phosphate unter verschiedenen Versuchsbedingungen. Das Verhalten der einzelnen Substrate des energieverteilenden Phosphokreatin-AdenylsäureSystems gleicht prinzipiell dem im linken Ventrikel beschriebenen (Abb. 8). Auffallend ist auch hier der hohe Phosphokreatingehalt im Myokard der prämedizierten Gruppe

Zur weiteren Klärung der Jodwirkung auf den Energieumsatz unter O2-Mangelsituation untersuchten wir das Verhalten der energiereichen Phosphatverbindungen (Voss et al. 1963). Das methodische Vorgehen wurde unverändert beibehalten. Die Bestimmung der Adenosin-Phosphatverbindungen erfolgte papierchromatographisch nach der Methode von Kunz, Schmid \& Siess (1958). Nach 3 Minuten Asphyxie fiel der ATP-Gehalt im Herzmuskel signifikant $a b$, während AMP und $P_{\mathbf{a}}$ anstiegen. Nach Vorbehandlung mit Jod war der ATP-Abfall im Myocard statistisch nicht mehr zu sichern. Entsprechend geringer war der Anstieg des anorganischen Phosphors. Zusammen mit VoGell untersuchten wir dann das Verhalten der Herzmuskelzelle, speziell der Mitochondrien und des Glykogens, unter asphyktischen Bedingungen im Experiment unter dem Elektronenmikroskop. Als Sitz der Enzyme für die Zellatmung, der oxydativen Phosphorylierung und des Krebs-Zyklus nehmen die Mitochondrien eine zen- 
trale Stellung im Stoffwechselgeschehen ein. Die Dichte der Mitochondrien ist der morphologische Ausdruck der Stoffwechselkapazität; sie sind im Papillarmuskel des Herzens besonders zahlreich vorhanden und weisen hier eine auffallende Dichte ihrer Cristae auf. Es ist daher zu erwarten, daß eine akute Hemmung des aeroben Stoffwechsels sich hier am ehesten und deutlichsten morphologisch manifestiert.

Die elektroenmikroskopischen Befunde zeigen, daß es schon nach einer kurzfristigen Asphyxie zu einem erheblichen Schwund der stoffwechselaktiven, proteingebundenen Glykogengranula kommt. Dieses Ergebnis stimmt mit dem biochemischen gut überein. Daneben führt eine kurze Asphyxie schon zu strukturellen Veränderungen, die hauptsächlich durch ein hypoxämisch bedingtes Odem hervorgerufen werden. Die morphologischen Veränderungen nehmen mit der Dauer der Asphyxie zu. Weniger empfindlich gegen den Sauerstoffmangel scheinen die Cristae mitochondrales zu sein. Eine Inaktivierung der SD verzögert sowohl den Glykogenschwund als auch die morphologisch faßbaren Veränderungen im Papillarmuskel nach Asphyxie.

Die Abbildungen 1 bis 5 sollen an Hand von elektronenmikroskopischen Fotos, die aus der Arbeit Vogell et al. (1964) entnommen wurden, unsere Ergebnisse veranschaulichen.

Durch Prämedikation der Versuchstiere mit anorganischem Jod und einem Pyrimido-pyrimidin-Derivat (Persantin ${ }^{\circledR}$, Dipyridamol, Hersteller: Dr. K. Thomae $\mathrm{GmbH}$, Biberach/Riss.) versuchten wir die Empfindlichkeit der Zelle gegenüber Sauerstoffmangel weiter herabzusetzen. Die Stoffwechselwirkung des Persantins bedarf noch weiterer Klärung. Sicher scheint sein peripherer Angriffspunkt zu sein, also im Bereich der Regelgröße. KunZ, ScHmm \& Siess (1962) sahen am isolierten Herzvorhofpräparat unter Persantin einen verzögerten Glykogen- und ATP-Abfall nach Sauerstoffentzug. Die Exgebnisse von Hochrein (1964), Hölscher (1965) und EkEström, PalÉus \& ÅBerg (1965) sind ähnlich. Thorn (1961) fand beim Sauerstoffmangel einen verzögerten Glykogenabbau nach Persantinzufuhr. GerLACH \& Deuticke (1963) führten die coronarerweiternde Wirkung des Präparats auf einen Anstau von Adenosin, einer energiearmen Abbaustufe des ATP, zurück (Abb. 6 und 7).

Die folgenden Untersuchungen dienten zur biochemischen Ergänzung der vorherigen Versuche (Voss \& Schoen 1965). Bei Hunden wurde durch Abklemmung der herznahen Vena cava superior et inferior und Vena azygos (inflow occlusion) eine Kreislaufunterbrechung herbeigeführt. Die Dauer einer tolerierten mittleren Kreislaufunterbrechung durch inflow occlusion beträgt beim Hund in Normothermie 8 bis 10 Minuten. 13 der 29 Versuchshunde erhielten folgende Prämedikation: 14 Stunden vor Versuchsbeginn $70 \mathrm{mg} / \mathrm{kg}$ Körpergewicht anorganisches Jod, 3 Stunden vor Beginn die gleiche Dosis und $1 / 2$ Stunde vor Beginn $10 \mathrm{mg} / \mathrm{kg}$ Persantin ${ }^{\otimes}$. Während des Experiments wurden $100 \mathrm{mg}$ Persantin in $500 \mathrm{ml}$ einer $5 \%$ igen Laevuloselösung appliziert. Die Dauer der Kreislaufunterbrechung wurde mit 10 Minuten festgesetzt. Danach wurden in der Muskulatur beider Herzkammern nach Entnahme mit Gefrierstop PKr, ATP, ADP, AMP, $\mathrm{P}_{\mathrm{a}}$ sowie BTS und MS bestimmt. Die Mittelwerte der einzelnen Versuchsgruppen wurden miteinander verglichen und die Signifikanz berechnet (WILCoxon-T'est).

Der Vergleich zwischen Kontrolltieren und unprämedizierten Tieren $(10 \mathrm{~min}$ 
Hypoxie) ergab folgende signifikante Unterschiede: Abfall des PKr und des ATP/ ADP-Quotienten, Anstieg der MS, des ADP, AMP und Lactat/Pyruvat-Quotienten. Dagegen brachte der Vergleich zwischen Kontrollen und prämedizierten Tieren lediglich einen signifikanten Anstieg von BTS und MS bei letzteren. Der entscheidende Vergleich zwischen Hypoxie-Tieren ohne und mit Prämedikation ergab einen signifikant

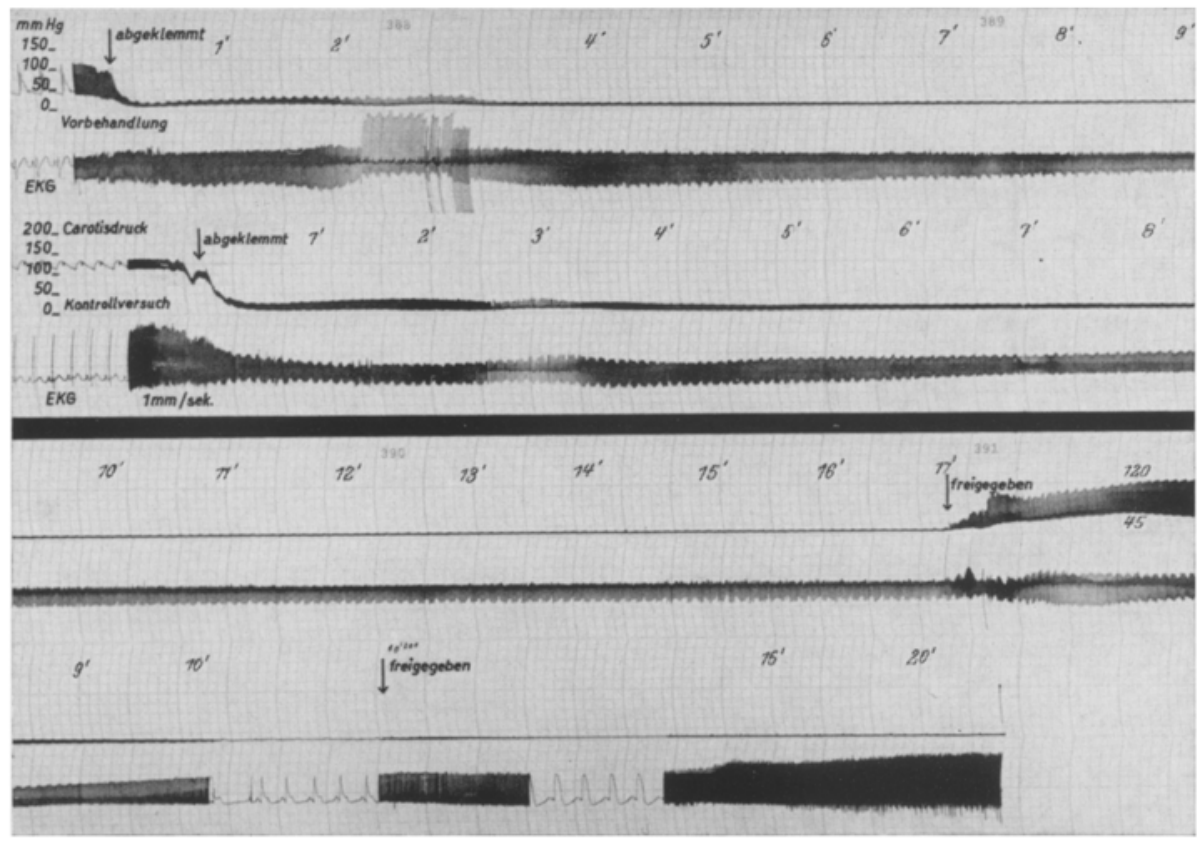

Abb. 10: Verhalten von EKG und Blutdruck nach inflow occlusion in Normothermie bei 2 Hunden. Die Kurve wurde ihrer Länge wegen halbiert: Der obere Teil zeigt den Verlauf bei einem prämedizierten Hund, der untere bei einem unprämedizierten. Registrierung mit Hellige-6-fach-Schreiber und Statham-Element. Der Blutdruck wurde blutig über einen in die linke Arteria femoralis eingelegten PVC-Katheter gemessen. Der arterielle Druck fallt nach der Kreislaufunterbrechung allmählich auf Werte nahe $0 \mathrm{mmHg}$ ab. Nach Wiederfreigabe des Kreislaufs nach 17 Minuten erfolgt prompt ein arterieller Drudkanstieg auf Werte über $100 \mathrm{mmHg}$, obwohl keine Herzmassage ausgeführt wurde. Beim unprämedizierten Hund (untere Kurve) kommt, obwohl der Kreislauf schon nach 10 Minuten wieder freigegeben wird, trotz nachweisbarer Herzaktion kein Druckanstieg zustande. Das Herz ist insuffizient geworden, die WBZ ist überschritten. (Nach Voss \& SCHOEN 1965)

höheren PKr-Gehalt und einen höheren ATP/ADP-Quotienten mit geringerem, hypoxaemisch bedingten Anstieg von $\mathrm{P}_{\mathrm{a}}, \mathrm{ADP}$ und MS bei den prämedizierten Hunden. Der MS/BTS-Quotient veränderte sich hier gegenüber den Kontrollen kaum. Man darf aus diesen Ergebnissen schließen, daß die Prämedikation dem Effekt der Hypoxie entgegenwirkt; denn es wurde keine Verschiebung zu energieärmeren Abbaustufen gefunden.

Die Dauer der tolerierten Kreislaufunterbrechung, bei der die Hälfte der Versuchstiere verstarb $\left(T_{50}\right)$, betrug bei nicht prämedizierten Hunden in Normothermie 
7,5 bis 8,0 Minuten, bei prämedizierten konnte sie auf $T_{50}=15$ Minuten, also auf das Doppelte ausgedehnt werden (Voss \& ScHoen 1965).

Wir hoffen, mit der Darlegung dieser Ergebnisse gezeigt zu haben, daß man durch geeignete pharmakologische Einflußnahme auf die regeltechnische Steuerung des Grundumsatzes die Wiederbelebungszeit verlängern kann.

\section{ZUSAMMENFASSUNG}

1. Zwischen der Länge der Wiederbelebungszeit eines Organs (Zeit vom Beginn einer Ischämie bis zum Auftreten irreversibler organischer Schädigungen) und der Höhe des Basalstoffwechsels besteht eine enge Korrelation. Daher hängt die Wiederbelebungszeit letztlich vom Blutspiegel der Schilddrüsenhormone ab.

2. Durch Drosselung der Schilddrüsenaktivität mit anorganischem Jod (Endojodin ${ }^{*}$ ) wurde bei normothermen Kaninchen und Hunden eine deutliche Verlängerung der WBZ nach Asphyxie und Hypoxie erzielt.

3. Die Jodwirkung wurde durch Hinzufügen eines Pyrimido-pyrimidin-Derivates (Persantin ${ }^{\circledR}$ ) noch verstärkt.

4. Es wurde festgestellt, daß nach kombinierter Applikation von Jod und Persantin der Abbau der energiereichen Phosphate Phosphokreatin und Adenosintriphosphat nach inflow occlusion im Herzmuskel und Gehirn langsamer erfolgte und der Anstieg von Lactat, Pyruvat und anorganischem Phosphor dementsprechend weniger steil war.

5. Morphologisch wurden im Papillarmuskel des Kaninchenherzens nach kombinierter Prämedikation unter dem Elektronenmikroskop geringer ausgeprägte Schäden gefunden, was auf eine verbesserte Energieausnutzung während der Anaerobiose oder Hypoxie zurückgeführt wird.

6. Die mittlere Dauer der Kreislaufunterbrechung mittels inflow occlusion konnte mit Hilfe der kombinierten Prämedikation (Jod und Persantin) bei Hunden in Normothermie von 8 auf 15 Minuten verlängert werden.

\section{ZITIERTE LITERATUR}

BRETSCHNEDER, H. J, 1961. Sauerstoffbedarf und -versorgung des Herzmuskels. Verh. dt. Ges. KreislForsch. 27, 32-59

- 1964. Uberlebenszeit und Wiederbelebungszeit des Herzens bei Normo- und Hyporhermie. Verh. dt. Ges. KreislForsch. 30, 11-34.

Ekeström, S., PalEus, S. \& Ånerg, T., 1965. Biochemische und physiologische Studien an anoxischen Hundeherzen mit extrakorporaler Zirkulation mit und ohne Zufuhr von Persantin ${ }^{\mathbb{B}}$. Cardiologia 46, 281-294.

Euler, C. von \& Holmgren, BJ., 1956. The thyroxine "receptor" of the thyroid-pituitary system. J. Physiol., Lond. 131, 125-146.

Gerlach, E. \& Deuticke, B., 1963. Bildung und Bedeutung von Adenosin in dem durch Sauerstoffmangel geschädigten Herzmuskel unter dem Einfluß von 2, 6-bis (diaethanolamino)-4,8-dipiperidinopyrimido-(5,4-d)-pyrimidin. Arzneimittel-Forsch. 13, 48-50. 
Hölscher, B., 1965. Untersuchungen zur Überlebenszeit des ischämischen Herzens in ihrer Bedeutung für die offene Herzchirurgie. Thoraxchirurgie 13, 44-55.

Hochrein, H., 1964. Uber den Energie-, Elektrolyt- und Enzymstoffwechsel des hypoxischen Herzens. Medsche Welt, Stuttg. Jg. 1964, 1112-1114.

Kunz, W., Schmid, W. \& Siess, M., 1958. Die Bestimmung von Adeninnucleotiden und Hexosephosphatestern in Gewebsextrakten. Z. ges. exp. Med. 130, 328-342.

- -1962 . Untersuchungen zur Wirkung von 2,6-bis(diaethanolamino)-4,8-dipiperidinopyrimido-(5,4-d)-pyrimidin auf den Herzstoffwechsel. Arzneimittel-Forsch. 12, 1098-1109.

Kuschinsky, G., 1933. Uber die Bedingungen der Sekretion des thyreotropen Hormons der Hypophyse. Arch. exp. Path. Pharmak. 170, 510-533.

Loeser, A., 1934. Die Umstimmung der Schilddrüsentätigkeit durch Jod. Klin. Wschr. 13, $533-534$.

Moore, D. H. \& Ruska, H., 1957. The structure of capillaries and small arteries. J. biophys. biochem. Cytol, 3, 457-462.

SCHARF, J. H., 1963. Experimentelle Voraussetzungen und theoretische Grundlagen zu einer antithyreoidalen Therapie unter Vermeidung zusätzlicher strumigener Effekte. Nova Acta Leopoldina, N.F. 26 ( $\mathrm{Nr} 163), 1-75$.

- Ehrentrand, F. \& Förster, W., 1954. Zytologische Untersuchungen über die Korrelationen $z$ wischen Hypophysenvorderlappen und Schilddrüse bei der Ratte unter Jodbelastung. Z. Zellforsch, mikrosk. Anat. 41, 132-171.

Schnerder, M., 1958. Uber die Wiederbelebung nach Kreislaufunterbrechung. Thoraxchirurgie 6, 95-106.

THORN, W., 1961. Metabolitkonzentrationen im Herzmuskel unter normalen, hypoxischen und anoxischen Bedingungen. Verh. dt. Ges. KreislForsch. 27, 76-90.

Vogell, W., Voss, R., SCHOEN, H. R, \& BeCKER, W. H., 1964. Elektronenmikroskopische und cytochemische Befunde am Papillarmuskel des Kaninchenherzens nach Sauerstoffentzug. Acta bistochem. 19, 234-248.

Voss, R. \& SCHoEN, H. R., 1965. Verlängerung der Kreislaufunterbrechung in Normothermie durch pharmakologische Vorbehandlung. Thoraxchirurgie 13, $29-44$.

- - \& Becker, W. H., 1964. Pharmakologische Möglichkeiten zur Verlängerung der Wiederbelebungszeit des Herzens nach Kreislaufunterbrechung in Normothermie. Thoraxchirurgie 11, 222-228.

- - Bettge, S. \& Becker, W. H., 1963. Der Einfluß von Hexamethyldiaminoisopropanoldijodid auf das Verhalten energiereicher Phosphatverbindungen in Herzmuskel und Gehirn nach Asphyxie. Z. ges. exp. Med. 137, 511-515.

\section{Diskussion im Anschluß an den Vortrag Voss \& Schoen}

MILleR: I understand that you attribute the increased hypoxic resistance of the heart of the treated animals to a lowering of the metabolism of the heart. Did you confirm this by measuring the $\mathrm{O}_{2}$-consumption of treated and untreated heart?

Voss: Das haben wir nicht gemacht.

SChARf: Zur Frage von Herrn Milier möchte ich bemerken, daß bei multilateraler Kontrolle biochemisch und morphologisch - auf die Bestimmung des $\mathrm{Q}_{2}$ verzichtet werden kann, obwohl natürlich im allgemeinen bei bekanntem $\mathrm{Q}_{2}$ die Befunde leichter überschaubar werden. 